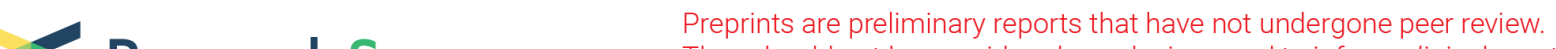

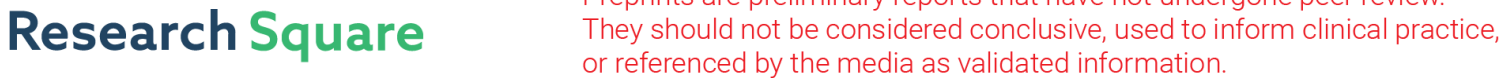

\section{ctDNA as A Biomarker of Progression in Oesophageal Adenocarcinoma}

\section{Vanessa F. Bonazzi ( $\nabla$ v.bonazzi@uq.edu.au )}

The University of Queensland https://orcid.org/0000-0002-2574-0334

\section{Lauren G. Aoude}

UQDI: The University of Queensland Diamantina Institute

\section{Sandra Brosda}

UQDI: The University of Queensland Diamantina Institute

James M. Lonie

UQDI: The University of Queensland Diamantina Institute

\section{Kalpana Patel}

UQDI: The University of Queensland Diamantina Institute

Julia J. Bradford

UQDI: The University of Queensland Diamantina Institute

\section{Lambros T. Koufariotis}

QIMR Berghofer: QIMR Berghofer Medical Research Institute

\section{Scott Wood}

QIMR Berghofer: QIMR Berghofer Medical Research Institute

\section{B. Mark Smithers}

PAH: Princess Alexandra Hospital

\section{Nicola Waddell}

QIMR Berghofer: QIMR Berghofer Medical Research Institute

\section{Andrew P. Barbour}

UQDI: The University of Queensland Diamantina Institute

\section{Research}

Keywords: ctDNA, sequencing, biomarker, oesophageal adenocarcinoma, survival, prognostic

Posted Date: November 16th, 2021

DOI: https://doi.org/10.21203/rs.3.rs-1041840/v1

License: (9) This work is licensed under a Creative Commons Attribution 4.0 International License. Read Full License 
Version of Record: A version of this preprint was published at ESMO Open on March 1st, 2022. See the published version at https://doi.org/10.1016/j.esmoop.2022.100452. 


\section{Abstract}

Background: The incidence of oesophageal adenocarcinoma (OAC) is rapidly increasing and despite improvements in treatment, the five-year survival rate remains poor. Prognostic biomarkers that address the genomic heterogeneity in this highly complex disease will aid the development of precision therapeutics and improve patient survival. The aim of this study was to determine whether circulating tumour DNA (ctDNA) has prognostic significance as a biomarker in OAC patients.

Methods: We profiled 209 blood and tumour samples from 57 OAC patients. Using a panel of 77 cancer genes, we sequenced ctDNA in plasma samples $(n=127)$ which were taken at multiple time points preand post-therapy. In parallel, we sequenced matched tumour samples from 39 patients using the same gene panel. To assess whether the ctDNA profile reflected the tumour heterogeneity, we sequenced additional multi-region primary tumour samples $(n=17)$. In addition, we analysed whole-genome and whole-exome sequencing data from primary tumours for a subset of 18 patients.

Results: Using a tumour agnostic approach, we found that detectable ctDNA variants in post-treatment plasma samples were associated with worse disease specific survival. To evaluate whether the ctDNA originated from the primary tumour, we performed a tumour informed analysis which confirmed posttreatment ctDNA variants were associated with worse survival. To determine whether ctDNA could be used as a clinical follow-up test, we assessed blood samples from multiple time points before and after treatment, in a subset of patients. Results showed that the variant allele frequency of ctDNA variants increased with disease recurrence.

Conclusion: This study demonstrates that ctDNA variants can be detected in patients with OAC and this has potential clinical utility as a prognostic biomarker for survival.

\section{Background}

Despite improvements in treatment regimens, the five-year survival of oesophageal adenocarcinoma (OAC) remains poor at 15\%-25\% $(1,2)$. For patients with curable (localized) disease, neoadjuvant therapy and surgical resection are the mainstays of treatment (3). Immune checkpoint blockade (ICB) has an increasing role in the adjuvant setting, currently for patients with residual disease after multimodality therapy (4). ICB also has an expanding role in the metastatic setting with chemotherapy $(5,6)$. The choice for therapy is currently based on OAC staging, which is limited to the TNM system, determined by endoscopy, endoscopic ultrasound and PET/CT scanning (7). Trials have shown that early PET response is a biomarker that can be used to tailor therapy, but more work is needed (8). A deeper understanding of the molecular biology of this highly complex disease will aid biomarker discovery leading to more novel, precision treatment delivery.

OAC tumours are heterogenous in their genomic composition (9). They are characterised by large scale re-arrangements such as chromothripsis, structural variations, copy number alterations and aneuploidy (9-17). A high mutation burden and chromosomal instability is a cause of intra-tumour heterogeneity 
(ITH) in OAC, both spatially and temporally (18-21). High levels of ITH result in clonal diversity which is a predictor of poor outcome and a cause of treatment failure in $\operatorname{OAC}(9,22-25)$.

Unravelling the complexities associated with tumour heterogeneity is a major challenge that is girt with difficulty. In addition to ITH, minimal residual disease (MRD) represents a significant problem in OAC with $50 \%$ of patients progressing after curative treatment(8). In an effort to tackle ITH and to assess MRD, genomic analyses of circulating tumour DNA (ctDNA) have been undertaken $(19,20,26-28)$. To date, studies in colorectal, urothelial, breast, and non-small cell lung cancer have reported prognostic value (29). However, studies from multiple cancer types, are confounded by additional ctDNA variants that are not tumour derived. Sequencing of peripheral blood cells is used to exclude variants classified as CHIP (clonal haematopoiesis of indeterminate potential) (30). While these methods improve the false-positive rates, the limitation is that variant calls are not confirmed as tumour derived. In OAC, there have been 4 small studies assessing the role of ctDNA as a biomarker of patient survival $(19,20,31,32)$. These studies vary in their methodology limiting their clinical utility. Additional OAC studies are required to implement the use of ctDNA as a diagnostic, predictive or prognostic tool in the clinic.

The primary aim of this study was to determine whether the ctDNA detected both pre- and/or posttreatment in plasma samples has prognostic or predictive significance in a cohort of OAC patients $(n=57)$. The second aim was to determine whether the ctDNA profile is reflective of ITH in these patients. The third aim was to determine the ability of ctDNA to detect disease recurrence during follow up. Using a commercially available CAPP-seq pan-cancer gene panel, we sequenced 183 tumour and plasma samples, which included multiple tumour biopsies for a subset of patients. Whole-genome sequencing or whole-exome sequencing data was available for an additional 24 tumour biopsies. This is the first study to describe the role of ITH in OAC and its impact on the development of ctDNA as a prognostic biomarker in patient treatment.

\section{Methods}

\section{Patient cohort}

In this retrospective study, we analysed plasma samples from 57 OAC patients treated at the Upper Gastro-Intestinal tract Clinic at the Princess Alexandra Hospital, Queensland, Australia, between September 2010 and October 2019. All participants provided written informed consent through the Cancer Evolution Biobank (HREC/10/PAH/152 and UQ/2011001287). Ethics approval for this project has been granted by the Metro South Human Research Ethics Committee (HREC/2019/QMS/55554), the University of Queensland Ethics Committee (2019002466) and QIMR Berghofer Human Research Ethics Committee (P3559). Clinical data including stage, tumour size, and therapy was collected for all participants (HREC/15/QPAH/614, Table 1, Sup Table 1).

Patients received standard of care which included endoscopy for diagnosis, perioperative chemotherapy or neo-adjuvant chemo-radiotherapy and surgery. The median age of onset of disease was 68 years (range 42 to 84 years). Patients recruited to this study ranged from stage I to III. The majority of patients 
were stage III, reflecting the patient population treated at the Princess Alexandra Hospital. The median PFS was 17.42 months (range, 1.9 to 92.5 months). The median DSS was 27.5 months (range, 5.1 to 92.5 months). Median follow up for survivors was 37 months (range 7.4 to 92.5 months) (Figure 1).

A baseline blood sample, described as B1, was taken at diagnosis and prior to treatment (Figure 1). An additional peri-operative blood (B2) was collected (Sup Table 1). Forty-six patients received neo-adjuvant chemo(radio)therapy. Twelve patients did not receive pre-operative chemotherapy or radiotherapy and went direct to surgery. For 10 patients, blood samples were taken at multiple time points during clinical follow up (Figure 2). In addition to their B1 and B2 blood samples, up to 2 post-therapy blood samples (B3 and B4) were taken, up to 60 months after diagnosis.

For a subset of patients $(n=39)$, tumour tissue was collected at endoscopy, prior to surgery. Biopsies were taken at several sites within the primary tumour and are described as T1, T2 and T3. Tissue was stored in RNAlater. DNA and RNA were extracted using the Qiagen AllPrep DNA/RNA mini kit according to the manufacturer's protocol (Qiagen, Germany).

\section{ctDNA extraction, amplification and analysis}

Blood samples for research were collected when patients underwent blood tests for standard of care. Plasma was isolated from either an Ariosa tube or EDTA tube. The Ariosa tube contains a preservation buffer and was processed within 3 days. The EDTA tubes were processed within 6 hours of collection. To ensure cellular debris did not contaminate downstream applications, an additional high g-force centrifugation step was performed after plasma isolation $\left(16000 \mathrm{xg}, 10\right.$ minutes at $\left.4^{\circ} \mathrm{C}\right)$.

For each patient, ctDNA was extracted from between $1.5 \mathrm{ml}$ and $5 \mathrm{ml}$ of plasma using the Avenio cfDNA Isolation kit (Roche Diagnostics, Risch-Rotkreuz, Switzerland), according to the manufacturer's protocol. The Isolation kit was used in conjunction with the AVENIO Expanded panel, a 77-gene panel associated with cancer diagnostics (33). Based on published SNVs associated with OAC, we estimated that the gene panel would identify ${ }^{3} 1$ mutation in at least $95 \%$ of patients (25). Final sequence libraries were quantified by Qubit fluorometer (Thermo Fisher Scientific, Waltham, MA) and profiled using a Bioanalyser (Agilent, Santa Clara, CA) (33).

Tumour library prep was performed using the AVENIO Tumor Expanded kit (Roche Diagnostics). We performed a buffer exchange which decreased the EDTA content in the input DNA. To do this, two ethanol washes were carried out using $3 x$ volume of clean up beads. DNA was then eluted in water for use with the kit. The DNA was sequenced according to the manufacturer's protocol.

The ctDNA and tumour libraries were sequenced using the Illumina HiSeq4000 to a minimum read depth of 500X. The average read depth was 5444.1 for the plasma samples (range 763 to 9265 ) and 1957.4 for the tumour samples (range 523 to 3836) (Sup Figure 1).

Variant analysis was performed using the AVENIO ctDNA Analysis software version 2. 


\section{Whole-genome and exome sequencing and analysis}

Whole-genome sequencing (WGS) was performed on matched tumour and buffy coat DNA from 11 patients. For 5 patients, WGS data was available for multiple biopsies where up to 3 tumour sites were assessed. A total of 17 biopsies were included in this study. The percent tumour content was assessed for each biopsy using Illumina SNP arrays and the qpure tool (34). All samples had tumour cellularity > $40 \%$. WGS was performed on the HiSeqX-ten (Illumina, San Diego CA, USA) with tumour DNA sequenced to a targeted read depth of $60 \mathrm{X}$ and the buffy coat to a read depth of 30X. The average read depth was 50.1 for the tumour samples (range 40 to 66 ) and 25.4 for the matched normal samples (range 22 to 30 ) (Sup Figure 1, Sup Table 1).

Whole-exome sequencing (WES) was available for 7 patients. Sequencing was performed on the HiSeqXten (Illumina, San Diego CA, USA) with tumour DNA sequenced to a targeted read depth of 100X and buffy coat to a read depth of 30X. The average read depth was 23.1 for the tumour samples (range 22 to 24) and 21.1 for the matched normal samples (range 13 to 26) (Sup Figure 1, Sup Table 1).

After sequencing the WGS and WES data underwent adaptor trimming with Cutadapt48 (version 1.9) and sequence reads were mapped to human reference genome GRCh37 using BWA-MEM49 (version 0.7.15) and SAMtools (version 1.9) (35). Duplicated reads were marked with Picard MarkDuplicates (version 2.8.15). The mean read depth was estimated for samples using qCoverage tool (http://sourceforge.net/projects/adamajava).

Germline and Somatic SNV and indel variants (using the matched tumour and control sample pair for a patient) were called using a dual calling strategy using qSNP (v2.1.4)(36) and GATK HaplotypeCaller (v3.8)(37). Germline and somatic variants were annotated with adamajava qannotate tool (v2.1.2) in SnpEff, hom and vcf2maf modes.

\section{Assessing called variants across multiple sequence data outputs for each patient}

For each patient, multiple sequence files (BAM files) were available including AVENIO Expanded panel data from plasma samples and/or tumour tissue and a subset of tumour tissue samples profiled with WGS or WES. To determine whether variants detected in the ctDNA samples were present in other sequenced samples from the same patient, a pileup method was used to determine the number of sequence reads containing a reference or variant base at each variant genomic position.

In the tumour agnostic approach, for each patient, sequence files from the B1 and B2 blood samples were aligned using the pile up method (Figure 1). A tumour informed approach was used to confirm whether ctDNA variants detected in plasma were present in the primary tumour. In this approach, BAM files from the AVENIO/WGS/WES were included in the pileup analysis. Pileups were generated using the AdamaJava tool qbasepileup v70 using pileup profile "DNA" and filtered with following parameters: CIGAR_M > 34, MD_mismatch <= 3, Flag_DuplicateRead == false, and MAPQ $>10$. 
Using the pileup output, sequencing data was filtered to remove germline variants, CHIP variants and false positive calls. Common SNVs present in ExAC or gnomAD databse (allele frequency of $>0.1 \%$ ) were excluded. SNVs present in the germline BAM files with $>20 \%$ of reads showing the variant were also excluded. Somatic variants with VAF $<0.001$ or fewer than 20 sequence reads were removed. Additionally, somatic variants with $<60 \%$ reads matched to the called base were removed. Variants that were present in $>2$ patients were also excluded.

For the tumour informed approach, variants were identified in tumour DNA using the AVENO platform or WGS/WES. The corresponding variant was then looked for in the ctDNA sequence reads. For a ctDNA variant to be considered tumour derived, it needed to match the tumour variant with minimum 10 reads in the ctDNA.

\section{Statistical analysis}

The primary aim of this study was to determine whether the ctDNA has prognostic or predictive significance in a cohort of OAC patients. Progression-free survival (PFS) was the time between surgery and first recurrence of metastatic disease. Disease specific survival (DSS) was the time from surgery until death from disease. A Log-rank (Mantel-Cox) test was used to assess ctDNA as a biomarker of survival in OAC (GraphPad Prism 8.3.1). The correlation between tumour regression, tumour variants and posttreatment blood variants was assessed using a chi-square test. For DSS data, hazard ratio (HR) was determined using a Cox proportional hazards regression model (R Foundation for Statistical Computing).

\section{Results}

ctDNA detection in stage I-III oesophageal adenocarcinoma using a tumour agnostic approach

We analysed plasma samples from a cohort of patients with OAC $(n=57)$ who were treated with curative intent (Figure 2). The clinicopathological characteristics are show in Table 1 and Supplementary Table 1.

To determine whether ctDNA isolated from both baseline (B1) and post-treatment (B2) plasma samples had prognostic significance, we used a tumour agnostic approach (Figure 1). This method gives insight into whether ctDNA can be a useful clinical tool when the patient tumour sample is not available for genomic reference. The median time between the B1 and B2 blood samples was 2.99 months (range 1 to 7 months).

Using a CAPP-seq 77 gene pan-cancer panel (33), we isolated, sequenced and analysed 114 plasma samples to detect tumour derived somatic ctDNA variants. We applied stringent filtering criteria to minimise the false-positive calls in the analysis. To eliminate CHIP variants and germline variants, we excluded variants with VAF $>0.4$. This filtering method is essential as CHIP variants are present in blood but are not tumour derived.

The median ctDNA concentration was $7.88 \mathrm{ng} / \mathrm{ml}$ (range 1.67 to $126.51 \mathrm{ng} / \mathrm{ml}$, Table 1 ) at baseline and $8.02 \mathrm{ng} / \mathrm{ml}$ (range 1.71 to $115 \mathrm{ng} / \mathrm{ml}$ ) in the post-treatment bloods. When we assessed the clinical 
features, we found no statistical association between ctDNA concentration and either clinical stage or pathological tumour size (data not shown). A pileup approach identified ctDNA variants in 22/57 patients (38\%). We detected a total of 90 ctDNA variants (range 0 to 7 per sample) occurring in the pre- and posttherapy plasma samples (Figure 3$)$. Recurrent variants $(n=14)$ between the baseline and post-treatment blood samples were observed in 12/57 (21\%) patients.

\section{ctDNA detection in stage $\mathrm{H}-\mathrm{III}$ oesophageal adenocarcinoma using a tumour informed approach}

Studies sequencing peripheral blood mononuclear cells (PBMCs) have shown CHIP variants may be detected in up to $23 \%$ of OAC patients (31). This method identifies CHIP variants so that they can be excluded from analysis, however, they cannot verify whether the remaining ctDNA variants are genuinely tumour derived. We used a tumour informed approach, profiling 185 matched plasma and tumour samples to determine whether ctDNA variants originated from the tumour sample.

Pre-treatment ctDNA and primary tumour samples from 39 patients were sequenced using the AVENIO pan-cancer 77 gene panel. We detected a total of 56 variants (median 2 per patient, range 1 to 9 ) in the primary tumour samples of 26/39 (67\%) patients (Figure 3$)$. In a subset of patients $(33 \%, n=13)$, no variants were detected in the tumour samples. For 17 patients, an additional biopsy from the same tumour was sequenced to assess the macroscopic tumour heterogeneity. The spatially distinct tumour biopsies (1 to $2 \mathrm{~cm}$ apart) are referred to as T1, T2, T3 (Figure 1). In 94\% (16/17) patients, where 2 tumour biopsies were sequenced with the AVENIO platform, we identified a variant in at least one of the tumour biopsies. In contrast, when only one tumour biopsy was sequenced, variants were observed in only $45 \%$ $(10 / 22)$ of patients. Sequencing 2 tumour biopsies per patient increased the likelihood of identifying variants that might otherwise be missed due to ITH.

WGS/WES data ( $\mathrm{n}=26)$ was available for 18 patients. WGS data was available for multiple tumour sites in 6/18 patients (Figure 4A, Supplementary Table 1). We focussed on the 77 genes that were represented on the AVENIO panel. For each patient, we performed a sequence pileup to compare the sequence data from the primary tumour with all available plasma sequence output. In total, we detected 68 tumour variants in WGS/WES, 22\% (15 variants) were aligned with ctDNA calls from the same patient (Figure 4AB). Patients with these variants $(n=10)$ are referred to as 'shedders' as there is evidence of the tumour shedding DNA into blood.

We assessed the concordance between the AVENIO platform and the WGS/WES data. The AVENIO platform provided data on a select panel of genes sequenced to a great depth (average 1955x for the tumour, average $5454 x$ for ctDNA, SupFigure 1 ). In contrast, WGS/WES provided sequence calls across the entire tumour genome but to a lower depth (average 50x). When we compared WGS/WES data to the AVENIO calls, $74 \%$ of the variants were in alignment (Figure 4A).

Patient SOG062 had two variants, in genes TP53 and KRAS, that were concordant between tumour regions and across WGS and AVENIO platforms (Figure 4A). Of note, only the KRAS variant was detected in the baseline blood. Similarly, SOG104, SOG179, SOG203 and SOG501 had concordant variants across 
platforms and multiple tumour regions. For SOG069, the AVENIO platform detected two distinct KDR variants which were not seen in the WGS data (Figure 4A) and were not detected in the plasma. Similarly, in SOG143, the AVENIO platform detected three variants, occurring in the tumour and the plasma, which were not observed in the WGS data.

Patient SOG066 and SOG083 had no tumour variant detected despite sequencing 2 tumour biopsies on each platform. The biopsies sequenced using WGS had high tumour content, tumour cellularity ranging from $47 \%$ to $60 \%$. The biopsies sequenced using the AVENIO platform had cellularity ranging from $22 \%$ to $60 \%$. Focussing on the 77 gene panel, there was an absence of variants in these cancer-related genes on both platforms, showing concordant results. Similarly, SOG506 had no variants detected using WES or the AVENIO platforms.

\section{OAC tumour heterogeneity}

To determine whether heterogeneity is an obstacle to the development of ctDNA as a clinical tool, we aligned the variant profile of patients with multiple tumour biopsies sequenced (Figure 4). Five patients were sequenced using the AVENIO capture alone. SOG427 had an RB1 variant which was only detected in one of the biopsies as well as in the baseline blood. In SOG415, T2 had three variants in TP53, PIK3CA and CDKN2A. Only two of these were seen in T1. However, all three variants were detected in the pre- and post-therapy blood samples. SOG443 (Figure 4) contained the highest number of variants and showed heterogeneity across both tumour and blood samples. Three variants were detected in T2 (genes BRCA1, PIK3CA and NTRK1). Only two of these were shared with T1. The pre-therapy blood was found to carry all three variants while the post-therapy blood only contained the NTRK1 variant. For 20 patients, we had multiple tumour biopsies sequenced using a combination of the AVENIO and WGS/WES platforms (Figure 4). $50 \%$ of patients (10/20) had concordant variants between the 2 biopsies. $40 \%$ of patients $(8 / 20)$ had individual variant specific to one biopsy. In two patients, no variant was observed.

The tumour from patient SOG143 was heterogeneous as T1 and T2 had a common PTCH1 variant but individual variants in BRCA2 and TP53 genes in T1 and T2, respectively (Figure 4). In the corresponding blood samples, only the PTCH1 variant was detected. The tumour from SOG221 also displayed heterogeneity. While the TP53 variant was seen in all biopsies analysed (across both platforms), the $R N F 43$ variant was only detected in 2 of 3 biopsies. None of these variants were observed in the blood samples.

\section{ctDNA-positivity as a prognostic biomarker}

It has been demonstrated that ctDNA from post-treatment bloods has prognostic value in the treatment of oesophageal cancer $(31,32)$. First, we sought to determine whether ctDNA positivity has prognostic value when applying the tumour agnostic approach. We analysed blood samples taken post-treatment, either after neoadjuvant therapy or surgery. We detected 35 variants (range 0 to 7 variants per patient, Figure 3 ) in the post-treatment blood samples. Univariate survival analyses demonstrated ctDNA positive patients had significantly worse DSS (median 58.8 months, range 7.4-92.5 months, * $\mathrm{p}=0.0130$, log rank, Figure 
5A) and a trend towards worse PFS (median 23 months, range 1.9 to 92.5 months, $p=0.1017$, log rank, Sup Figure 2) compared with ctDNA negative patients (median DSS not reached, range 5.1 to 80.8 months and median PFS not reached, range 2.1 to 80.8 months). There was no association between baseline blood samples and PFS or DSS (data not shown).

Various ctDNA studies have used a cut-off of 2 variants to be classified ctDNA positive $(31,38)$. We repeated the survival analysis splitting the cohort into 2 groups: patients with 0 or 1 variant versus patients with $>1$ variant and the outcome remained significant (median 13.5, range 7.8 to 90.5 months, ${ }^{*} p=0.0205$, log rank, Supplementary Figure 2). Considering 3 categories: 0 variant, 1 variant, and $>1$ variant, a clear separation was observed between the groups ( $\left.{ }^{*} \mathrm{p}=0.0175\right)$. In our data, patients with at least 1 ctDNA variant had worse DSS than the 0 variants group, therefore patients with a minimum of 1 variant were considered ctDNA positive.

We assessed whether the number of ctDNA variants was associated with the pathological tumour size. We found that patients with small tumours, $<10 \mathrm{~mm}$, were less likely to have detectable variants in their post-treatment plasma. However, this did not reach significance $(p=0.1211$, Sup Figure 2$)$. We next examined whether tumour regression was associated with the number of ctDNA variants. We compared patients that were ctDNA positive to those that were negative. Patients with major histological response (90-100\% tumour regression) were more likely to be ctDNA negative $(p=0.0556$, chi-square test, Figure 5B).

We then sought to determine whether the tumour informed approach to ctDNA detection would have prognostic significance. We analysed blood samples taken during the peri-operative period, either after neoadjuvant therapy or surgery. Patients with detectable variants in their tumour $(n=30)$ were split into 2 groups, shedders and non-shedders, where shedders had ctDNA variants verified by the primary tumour genomic profile. A third group was defined as patients with no variant detected in their tumour, $n=16$ (Supplementary Figure 3). The non-shedders and the patients with no variant detected were pooled as univariable survival analysis indicated they had the same DSS (median survival not reached, range 5.192.6 months, $p=N S$, log rank). We examined the post-treatment bloods and found that shedders had worse DSS (median survival 10.6 months, range 7.99-37.55 months, *** $\mathrm{p}=0.0007$, log rank, Figure 5C) and worse PFS (median survival 9.27 months, range 2.1-37.55 months, * $\mathrm{p}=0.0311$, log rank, Supplementary Figure 3 ). There was no association between baseline blood samples and survival (data not shown).

We next assessed whether the presence of the primary tumour at the peri-operative time point influenced the ability to detect ctDNA variants. When patients had a complete response and the tumour was absent at blood collection, there was no significant correlation between shedders and non-shedders/no variant detected (Supplementary Figure 3). However, when the tumour was present, shedders had worse DSS $\left({ }^{*} \mathrm{p}=0.0473, \log\right.$ rank, Figure 5D).

ctDNA detection during following treatment and disease recurrence

Page 10/25 
For 10 patients, we analysed multiple blood samples, one baseline sample and several post-treatment blood samples (Figure 2). Five patients were alive with no sign of recurrence (NSR). Of this group, 3 patients had no variant detected in any of their blood samples using the AVENIO platform (Figure 6). SOG062, the longest survivor, had no variant above the threshold for calling in the post-treatment blood samples, however, the KRAS variant identified by WGS and AVENIO tumour sequencing was detected with a low number of reads in the plasma. The KRAS variant was higher at the time of diagnosis. Post chemotherapy, the VAF reduced but was still detectable. After surgery, at 60 months from diagnosis, the $K R A S$ variant was present but remained below the threshold for calling, in this long-term survivor. SOG315 had an $A P C$ variant at baseline. At all post-treatment time points, this variant was detectable at low levels, below the threshold for calling. In the blood sample taken after surgery, 21 months from diagnosis, a TP53 variant was identified at low levels. Neither variant was confirmed in the primary tumour, however only one tumour biopsy was available for sequencing.

Three patients were alive with disease. SOG425 had 6 variants detected in the blood at baseline. None of these variants increased in the post-treatment samples, however the longest time point available was only 1 month after resection. Similarly, SOG460 had very short follow-up time points, however, a MET variant was detectable in all samples at low levels. In SOG317, we observed a PIK3CA variant present at baseline and post-treatment blood samples. The VAF increased at 21-months post-surgery reflecting the disease progression which was confirmed by CT scan. Two patients died of their disease (SOG490 and SOG529). Both patients presented low levels of ctDNA variants in their baseline blood sample. However, post-treatment, high levels of several ctDNA variants were detected in their blood (Figure 6).

\section{Discussion}

Here, we present a study examining the role of ctDNA as a prognostic biomarker in patients with resectable OAC. We assessed ctDNA isolated from both pre- and post-treatment plasma samples in a cohort of 57 patients treated with curative intent. Additionally, we sequenced tumour samples from 46 patients and determined whether their ctDNA profile was reflective of the tumour genomic heterogeneity.

We performed ctDNA sequencing using a commercially available kit targeting 77 cancer genes. When aligned with WGS data from the matched tumour, we detected germline variants with high accuracy (data not shown) indicating the kit was able to identify variants across the whole gene panel. While the panel provided very high-depth sequence reads, it is limited to a panel of 77 cancer associated genes. In OAC, while there are frequently mutated genes, somatic mutations occur across the genome with no specific hotspot $(9,39)$, meaning that variants could have been missed with the non-OAC specific panel.

To more accurately filter the sequence data, other studies have used patient PBMCs to eliminate CHIP variants $(31,32)$. The lack of primary tumour genomic sequence data is a limitation of these studies as acknowledged by the authors themselves (29). No standardised method exists to remove false-positive variants identified in ctDNA. In our study, we hypothesized that focusing on a tumour informed approach 
would give more accurate results when assessing ctDNA variants extracted from plasma samples in OAC patients.

Tumour genomic heterogeneity is a well described characteristic of $\operatorname{OAC}(9,39)$. This key genomic feature impacts the utility of ctDNA variants as biomarkers for clinical diagnosis. We are the first to compare ctDNA variants detected in blood samples with multiple tumour biopsies from the same primary tumour. We showed that when only one tumour biopsy was sequenced, only few variants were identified in both ctDNA and the tumour, most likely due to ITH. When we sequenced multiple biopsies from each patient, there was much more concordance between the tumour and ctDNA genomic profiles. This finding is in line with Maron et al. who showed only $26 \%$ concordance between the genomic profile of primary tumours, metastatic tumours and ctDNA in gastroesophageal adenocarcinoma(19). This suggests that tumours may not shed ctDNA homogenously across the tumour mass, and raises the question of whether only certain sub-clones shed ctDNA.

TP53 is the most frequently mutated gene in OAC with mutations found across the length of the gene( 12 , 15). Mutations occur in approximately $80 \%$ of primary tumours. Sporadic loss-of-function mutations have also been reported in other tumour suppressor genes (CDKN2A, ARID1A and SMAD4) $(15,40)$. Furthermore, variants in TP53 are problematic for ctDNA studies in OAC as CHIP variants frequently occur in that locus(31). In our study, TP53 was the most frequently mutated gene in patients with detectable variants (45\%) in the filtered data. This is in alignment with a previous ctDNA study detecting TP53 in $50 \%$ of patients(31). This frequency is lower than expected and this may be due to a combination of factors such as ITH and non-uniform DNA shedding across the tumour. Furthermore, it has been shown that in other hypermutated cancers, such as non-small cell lung cancer, as few as $10 \%$ of ctDNA variants matched the tumour of origin(30).

In previous ctDNA studies, there are inconsistencies in the definition of ctDNA positivity with a cut-off of either 1 or 2 variants $(31,32,38)$. In our study, the detection of a 1 or 2 ctDNA variants was significantly associated with DSS. Our tumour agnostic approach showed that the presence of a ctDNA variant after therapy was associated with worse DSS. One of the challenges of this approach is that ctDNA variants were not verified as specifically originating from the primary tumour. This may pose a problem if this approach is to be implemented in the clinical decision process. To address this, we performed a tumour informed analysis which confirmed ctDNA has prognostic significance. Furthermore, assessing the baseline blood samples using the tumour informed approach, we observed that ctDNA in the baseline samples is not sufficient to discriminate patient prognosis. Even if ctDNA is detected at the baseline, it does not indicate a palliative approach is appropriate, as patient may still respond to treatment.

To determine whether serial time point analysis of ctDNA variants had prognostic significance, we assessed blood samples from multiple time points in 10 patients. Of the 5 patients with no sign of recurrence, three had no ctDNA variants identified, the other 2 patients had very low levels of ctDNA below the threshold for calling in their most recent blood sample. Using our pileup approach for sequencing analysis, we were able to detect these low-level variants. We hypothesise that the patient immune system 
has eliminated most of the tumour cells and these ctDNA variants may be good individual prognostic markers of disease. Long term, these patients may benefit from close monitoring to anticipate disease recurrence.

Three patients had progressive disease. ctDNA variants were detected in the most recent blood sample in two of these three patients. For the third patient, SOG425, the baseline blood sample revealed very high ctDNA variant levels before chemoradiotherapy. After treatment, the ctDNA levels dropped. However, the final sample collection timepoint was less than 1-month post-surgery which limited the follow-up analysis. Tracking these variants at a later timepoint may have more clinical relevance in terms of disease progression.

The two patients who died from their disease (SOG490 and SOG529) had ctDNA variants with increasing VAF in their post-treatment blood samples. We hypothesise that these patients had more aggressive disease reflected in their ctDNA profile.

This study provides a strong argument for the use of ctDNA as a clinical tool. It has the potential to serve as a prognostic test after neoadjuvant chemo/radiotherapy and may inform treatment choices before and after surgery. ctDNA may also represent a viable liquid biopsy, for shedders specifically. Indeed, these patients have the worst outcomes and it is of particular importance to improve prognostication and treatment options for this group. There is a need for standardisation of ctDNA quantification methods and timepoints to apply these findings to the clinic. In clinical trials, ctDNA positive patients may be the ideal candidates for post-operative immunotherapy. In CheckMate 577 (4), residual disease patients benefited from immunotherapy, perhaps those with good response and positive ctDNA should also be considered for therapy.

\section{Conclusion}

The results of this study have shown that ctDNA analysis can reflect the genomic heterogeneity associated with OAC tumours. ctDNA assessment can be used as a personalised prognostic biomarker for patients with OAC. To be implemented in future clinical assessment, a robust pipeline for ctDNA analysis needs to be developed in large clinical studies.

\section{Abbreviations}

AJCC American Joint Committee on Cancer

CHIP clonal hematopoiesis of indeterminate potential

ctDNA circulating tumour DNA

CXRT chemoradiotherapy

DSS disease-free survival 
ICB immune checkpoint blockade

ITH intra-tumour heterogeneity

MRD minimal residual disease

NSR no sign of recurrence

OAC oesophageal adenocarcinoma

PBMC peripheral blood mononuclear cells

PFS progression-free survival

SNV single nucleotide variant

VAF variant allele frequency

WES whole-exome sequencing

WGS whole-genome sequencing

\section{Declarations}

Ethics approval and consent to participate: All participants provided written informed consent through the Cancer Evolution Biobank (HREC/10/PAH/152 and UQ/2011001287). Ethics approval for this project has been granted by the Metro South Human Research Ethics Committee (HREC/2019/QMS/55554), the University of Queensland Ethics Committee (2019002466) and QIMR Berghofer Human Research Ethics Committee (P3559). Clinical data including stage, tumour size, and therapy was collected for all participants (HREC/15/QPAH/614).

Consent for publication: Not applicable

Availability of data and materials (Due to the recently imposed file size restrictions by EGA, our data upload has been significantly delayed. We will update you as soon as we get notification from EGA): The data that support the findings of this study are available in the European Genome-phenome Archive, study ID EGAS00001002864, data set ID .... Additional data are available through study ID ..., data set ID... EGA identifiers can be found in Supplementary Table 1.

Competing interests: AB received a Roche Investigator Initiated clinical research grant. NW is a co-founder and board member of genomiQa. All other authors declare no potential conflicts of interest.

Funding: Support was provided by the Randall Foundation and a Metro South Health Research Support Scheme program grant. LGA is supported by a National Health and Medical Research Council of Australia (NHMRC) Early Career Fellowship (1109048). NW is supported by an NHMRC Senior Research Fellowship 
(1139071). The Cancer Evolution Biobank is supported by the PA Research Foundation. This research was carried out at the Translational Research Institute, Woolloongabba, QLD 4102, Australia. The Translational Research Institute is supported by a grant from the Australian Government.

\section{Authors' contributions:}

VFB, LGA, SB and APB contributed to the study design, methodology and data analysis. APB and BMS recruited the patients. $\mathrm{KP}, \mathrm{JML}$ and JJB performed laboratory experiments related to tissue and blood samples. VFB and LGA performed all the specific ctDNA experiments and wrote the manuscript. SB, LTK, SW and NW contributed to the acquisition and analysis of whole- genome and whole-exome data. All authors edited, read and approved the final manuscript.

Acknowledgements: We acknowledge the support of the Estate of the late Alec Pearman and Ms Di Jameson.

\section{References}

1. Thrift AP. The epidemic of oesophageal carcinoma: Where are we now? Cancer Epidemiol. 2016;41:88-95.

2. Smyth EC, Lagergren J, Fitzgerald RC, Lordick F, Shah MA, Lagergren P, et al. Oesophageal cancer. Nat Rev Dis Primers. 2017;3:17048.

3. Lordick F, Mariette C, Haustermans K, Obermannova R, Arnold D, Committee EG. Oesophageal cancer: ESMO Clinical Practice Guidelines for diagnosis, treatment and follow-up. Ann Oncol. 2016;27(suppl 5):v50-v7.

4. Kelly RJ, Ajani JA, Kuzdzal J, Zander T, Van Cutsem E, Piessen G, et al. Adjuvant Nivolumab in Resected Esophageal or Gastroesophageal Junction Cancer. N Engl J Med. 2021;384(13):1191-203.

5. Kojima T, Shah MA, Muro K, Francois E, Adenis A, Hsu CH, et al. Randomized Phase III KEYNOTE-181 Study of Pembrolizumab Versus Chemotherapy in Advanced Esophageal Cancer. J Clin Oncol. 2020;38(35):4138-48.

6. Shah MA, Kojima T, Hochhauser D, Enzinger P, Raimbourg J, Hollebecque A, et al. Efficacy and Safety of Pembrolizumab for Heavily Pretreated Patients With Advanced, Metastatic Adenocarcinoma or Squamous Cell Carcinoma of the Esophagus: The Phase 2 KEYNOTE-180 Study. JAMA Oncol. 2019;5(4):546-50.

7. Amin MB, Greene FL, Edge SB, Compton CC, Gershenwald JE, Brookland RK, et al. The Eighth Edition AJCC Cancer Staging Manual: Continuing to build a bridge from a population-based to a more "personalized" approach to cancer staging. CA Cancer J Clin. 2017;67(2):93-9.

8. Barbour AP, Walpole ET, Mai GT, Barnes EH, Watson DI, Ackland SP, et al. Preoperative cisplatin, fluorouracil, and docetaxel with or without radiotherapy after poor early response to cisplatin and fluorouracil for resectable oesophageal adenocarcinoma (AGITG DOCTOR): results from a multicentre, randomised controlled phase II trial. Ann Oncol. 2020;31(2):236-45. 
9. Secrier M, Li X, de Silva N, Eldridge MD, Contino G, Bornschein J, et al. Mutational signatures in esophageal adenocarcinoma define etiologically distinct subgroups with therapeutic relevance. Nat Genet. 2016;48(10):1131-41.

10. Alexandrov LB, Nik-Zainal S, Wedge DC, Aparicio SA, Behjati S, Biankin AV, et al. Signatures of mutational processes in human cancer. Nature. 2013;500(7463):415-21.

11. Chang K, Creighton CJ, Davis C, Donehower L, Drummond J, Wheeler D, et al. The Cancer Genome Atlas Pan-Cancer analysis project. Nature Genetics. 2013;45(10):1113-20.

12. Dulak AM, Stojanov P, Peng S, Lawrence MS, Fox C, Stewart C, et al. Exome and whole-genome sequencing of esophageal adenocarcinoma identifies recurrent driver events and mutational complexity. Nat Genet. 2013;45(5):478-86.

13. Findlay JM, Castro-Giner F, Makino S, Rayner E, Kartsonaki C, Cross W, et al. Differential clonal evolution in oesophageal cancers in response to neo-adjuvant chemotherapy. Nat Commun. 2016;7:11111.

14. Frankel A, Armour N, Nancarrow D, Krause L, Hayward N, Lampe G, et al. Genome-wide analysis of esophageal adenocarcinoma yields specific copy number aberrations that correlate with prognosis. Genes Chromosomes Cancer. 2014;53(4):324-38.

15. Nones K, Waddell N, Wayte N, Patch AM, Bailey P, Newell F, et al. Genomic catastrophes frequently arise in esophageal adenocarcinoma and drive tumorigenesis. Nat Commun. 2014;5:5224.

16. Noorani A, Bornschein J, Lynch AG, Secrier M, Achilleos A, Eldridge M, et al. A comparative analysis of whole genome sequencing of esophageal adenocarcinoma pre- and post-chemotherapy. Genome Res. 2017;27(6):902-12.

17. Wang K, Johnson A, Ali SM, Klempner SJ, Bekaii-Saab T, Vacirca JL, et al. Comprehensive Genomic Profiling of Advanced Esophageal Squamous Cell Carcinomas and Esophageal Adenocarcinomas Reveals Similarities and Differences. Oncologist. 2015;20(10):1132-9.

18. Burrell RA, McGranahan N, Bartek J, Swanton C. The causes and consequences of genetic heterogeneity in cancer evolution. Nature. 2013;501(7467):338-45.

19. Maron SB, Chase LM, Lomnicki S, Kochanny S, Moore KL, Joshi SS, et al. Circulating Tumor DNA Sequencing Analysis of Gastroesophageal Adenocarcinoma. Clin Cancer Res. 2019;25(23):7098-112.

20. Pectasides E, Stachler MD, Derks S, Liu Y, Maron S, Islam M, et al. Genomic Heterogeneity as a Barrier to Precision Medicine in Gastroesophageal Adenocarcinoma. Cancer Discov. 2018;8(1):37-48.

21. Murugaesu N, Wilson GA, Birkbak NJ, Watkins T, McGranahan N, Kumar S, et al. Tracking the genomic evolution of esophageal adenocarcinoma through neoadjuvant chemotherapy. Cancer Discov. 2015;5(8):821-31.

22. Martinez P, Timmer MR, Lau CT, Calpe S, Sancho-Serra Mdel C, Straub D, et al. Dynamic clonal equilibrium and predetermined cancer risk in Barrett's oesophagus. Nat Commun. 2016;7:12158.

23. Ross-Innes CS, Becq J, Warren A, Cheetham RK, Northen H, O'Donovan M, et al. Whole-genome sequencing provides new insights into the clonal architecture of Barrett's esophagus and esophageal adenocarcinoma. Nat Genet. 2015;47(9):1038-46. 
24. Zhang J, Fujimoto J, Zhang J, Wedge DC, Song X, Zhang J, et al. Intratumor heterogeneity in localized lung adenocarcinomas delineated by multiregion sequencing. Science. 2014;346(6206):256-9.

25. Frankell AM, Jammula S, Li X, Contino G, Killcoyne S, Abbas $S$, et al. The landscape of selection in 551 esophageal adenocarcinomas defines genomic biomarkers for the clinic. Nat Genet. 2019;51(3):506-16.

26. Lebofsky R, Decraene C, Bernard V, Kamal M, Blin A, Leroy Q, et al. Circulating tumor DNA as a noninvasive substitute to metastasis biopsy for tumor genotyping and personalized medicine in a prospective trial across all tumor types. Mol Oncol. 2015;9(4):783-90.

27. Wan R, Wang Z, Lee JJ, Wang S, Li Q, Tang F, et al. Comprehensive Analysis of the Discordance of EGFR Mutation Status between Tumor Tissues and Matched Circulating Tumor DNA in Advanced Non-Small Cell Lung Cancer. J Thorac Oncol. 2017;12(9):1376-87.

28. Wyatt AW, Annala M, Aggarwal R, Beja K, Feng F, Youngren J, et al. Concordance of Circulating Tumor DNA and Matched Metastatic Tissue Biopsy in Prostate Cancer. J Natl Cancer Inst. 2017;109(12).

29. De Paula B, E CS. Personalising care in oesophageal cancer care with liquid biopsy. Br J Cancer. 2021.

30. Razavi P, Li BT, Brown DN, Jung B, Hubbell E, Shen R, et al. High-intensity sequencing reveals the sources of plasma circulating cell-free DNA variants. Nat Med. 2019;25(12):1928-37.

31. Ococks E, Frankell AM, Masque Soler N, Grehan N, Northrop A, Coles H, et al. Longitudinal tracking of 97 esophageal adenocarcinomas using liquid biopsy sampling. Ann Oncol. 2021;32(4):522-32.

32. Azad TD, Chaudhuri AA, Fang P, Qiao Y, Esfahani MS, Chabon JJ, et al. Circulating Tumor DNA Analysis for Detection of Minimal Residual Disease After Chemoradiotherapy for Localized Esophageal Cancer. Gastroenterology. 2020;158(3):494-505 e6.

33. Jiang J, Adams HP, Yao L, Yaung S, Lal P, Balasubramanyam A, et al. Concordance of Genomic Alterations by Next-Generation Sequencing in Tumor Tissue versus Cell-Free DNA in Stage I-IV NonSmall Cell Lung Cancer. J Mol Diagn. 2020;22(2):228-35.

34. Song S, Nones K, Miller D, Harliwong I, Kassahn KS, Pinese M, et al. qpure: A tool to estimate tumor cellularity from genome-wide single-nucleotide polymorphism profiles. PLoS One. 2012;7(9):e45835.

35. Li H, Handsaker B, Wysoker A, Fennell T, Ruan J, Homer N, et al. The Sequence Alignment/Map format and SAMtools. Bioinformatics. 2009;25(16):2078-9.

36. Kassahn KS, Holmes O, Nones K, Patch AM, Miller DK, Christ AN, et al. Somatic point mutation calling in low cellularity tumors. PLoS One. 2013;8(11):e74380.

37. McKenna A, Hanna M, Banks E, Sivachenko A, Cibulskis K, Kernytsky A, et al. The Genome Analysis Toolkit: a MapReduce framework for analyzing next-generation DNA sequencing data. Genome Res. 2010;20(9):1297-303.

38. Abbosh C, Birkbak NJ, Wilson GA, Jamal-Hanjani M, Constantin T, Salari R, et al. Phylogenetic ctDNA analysis depicts early-stage lung cancer evolution. Nature. 2017;545(7655):446-51. 
39. Nones K, Waddell N, Wayte N, Patch AM, Bailey P, Newell F, et al. Genomic catastrophes frequently arise in esophageal adenocarcinoma and drive tumorigenesis. Nat Commun. 2014;5:5224.

40. Weaver JMJ, Ross-Innes CS, Shannon N, Lynch AG, Forshew T, Barbera M, et al. Ordering of mutations in preinvasive disease stages of esophageal carcinogenesis. Nat Genet. 2014;46(8):83743.

\section{Tables}

Table 1. Patient characteristics. Clinically relevant characteristics include age, gender, stage, tumour size and type of treatment. Patient survival is indicated in months. AJCC is the American Joint Committee on Cancer. 


\begin{tabular}{|c|c|c|}
\hline Variables & Number of patients $(n=57)$ & $\begin{array}{l}\text { Percent } \\
\text { (\%) }\end{array}$ \\
\hline \multicolumn{3}{|l|}{ Age at diagnosis (years) } \\
\hline$<60$ & 16 (range 42-59) & 28.1 \\
\hline$\geq 60$ & 41 (range 60-85) & 71.9 \\
\hline \multicolumn{3}{|l|}{ Gender } \\
\hline Male & 53 & 93.0 \\
\hline Female & 4 & 7.0 \\
\hline \multicolumn{3}{|c|}{ Clinical Stage (AJCC 8th edition) } \\
\hline Stage I & 7 & 12.3 \\
\hline Stage II & 12 & 21.1 \\
\hline Stage III & 38 & 66.7 \\
\hline \multicolumn{3}{|c|}{ Median pathological tumour size (mm) } \\
\hline$<10$ & 20 (range 0 to 4 ) & 35.1 \\
\hline 10 to 40 & 25 (range 12 to 35 ) & 43.9 \\
\hline$>40$ & 12 (range 45 to 80 ) & 21.1 \\
\hline \multicolumn{3}{|l|}{ PFS } \\
\hline$<12$ months & 15 (range 1.9 to 11.9 ) & 26.3 \\
\hline$>12$ months & 42 (range 12 to 92.5 ) & 73.7 \\
\hline \multicolumn{3}{|l|}{ DSS } \\
\hline$<24$ months & 25 (range 5.1 to 21.7 ) & 43.9 \\
\hline$>24$ months & 32 (range 25 to 92.5 ) & 56.1 \\
\hline \multicolumn{3}{|l|}{ Neo-adjuvant treatment } \\
\hline Direct to surgery & 12 & 21.1 \\
\hline Pre-operative CXRT & 26 & 45.6 \\
\hline Pre-operative Chemotherapy & 19 & 33.3 \\
\hline
\end{tabular}

Figures 


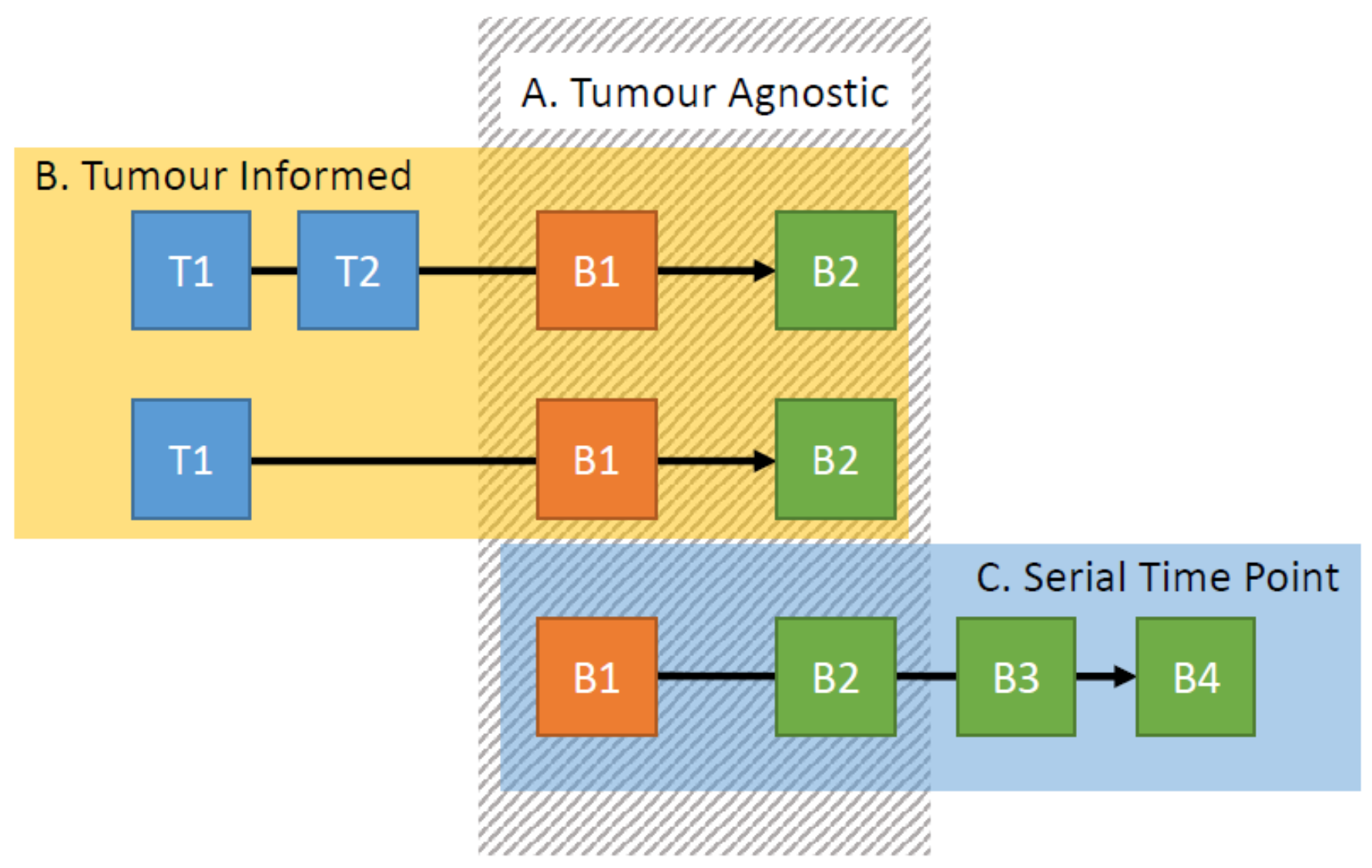

\section{Figure 1}

Study design. A. Tumour Agnostic analysis on ctDNA variants from Baseline (B1, orange squares) and post-treatment samples (B2, green squares). B. Tumour informed approach aligning the ctDNA variants to matched primary tumour samples (T1). For a subset of patients, an additional biopsy (T2) was taken from a different site within the one primary tumour. In the tumour informed approach, a ctDNA variant was only considered positive if it was present in a matching primary tumour sample. C. Analysis of ctDNA at multiple time points post-treatment (B3 and B4). Blue squares represent pre-treatment tumour biopsies. 


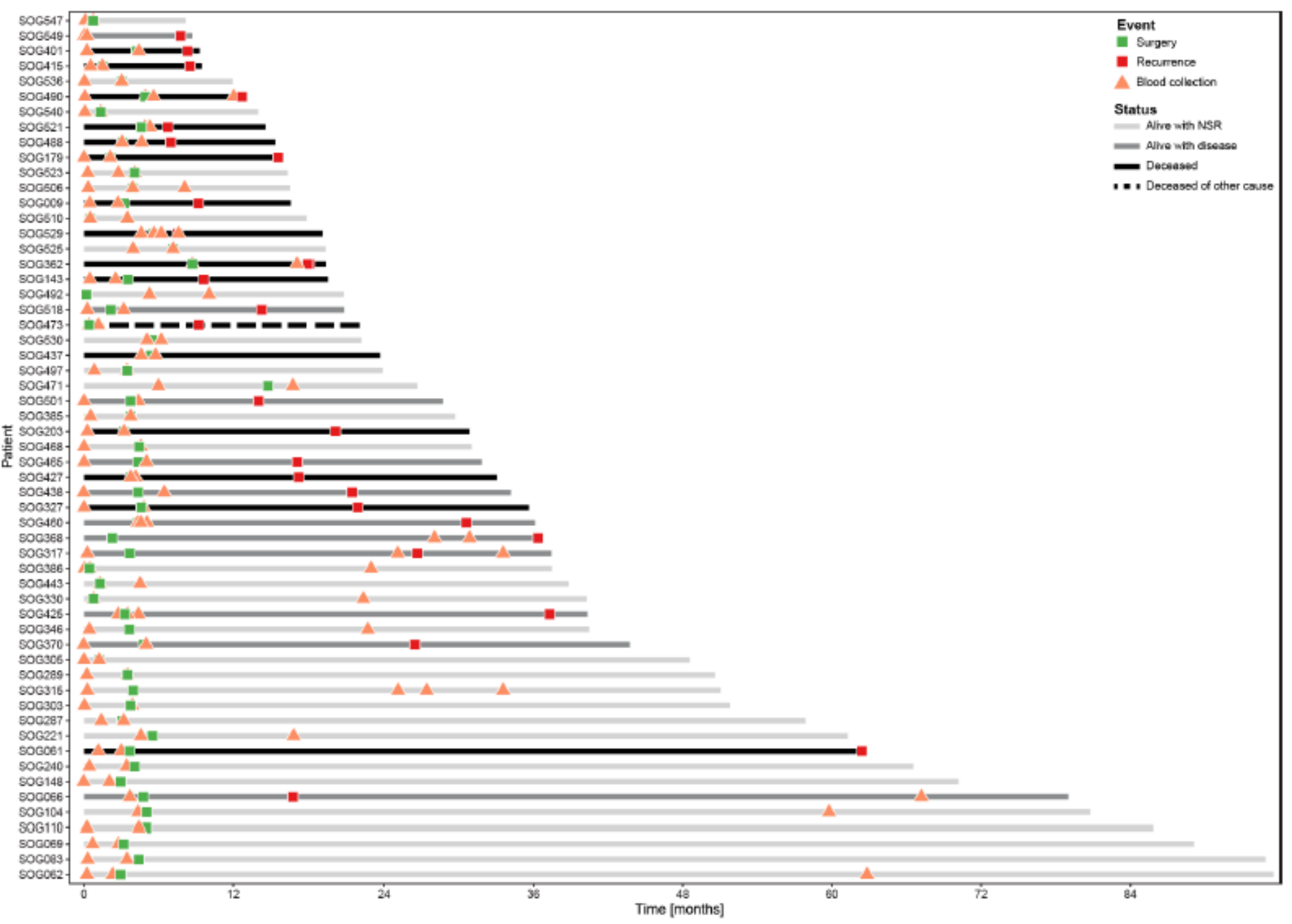

Figure 2

Swimmer plot. The clinical timeline is shown for each patient. Triangles indicate the blood collection timepoints. Where possible, tumours were sampled at time of diagnosis. The colour of each bar represents the disease specific survival. NSR is no sign of recurrence. 


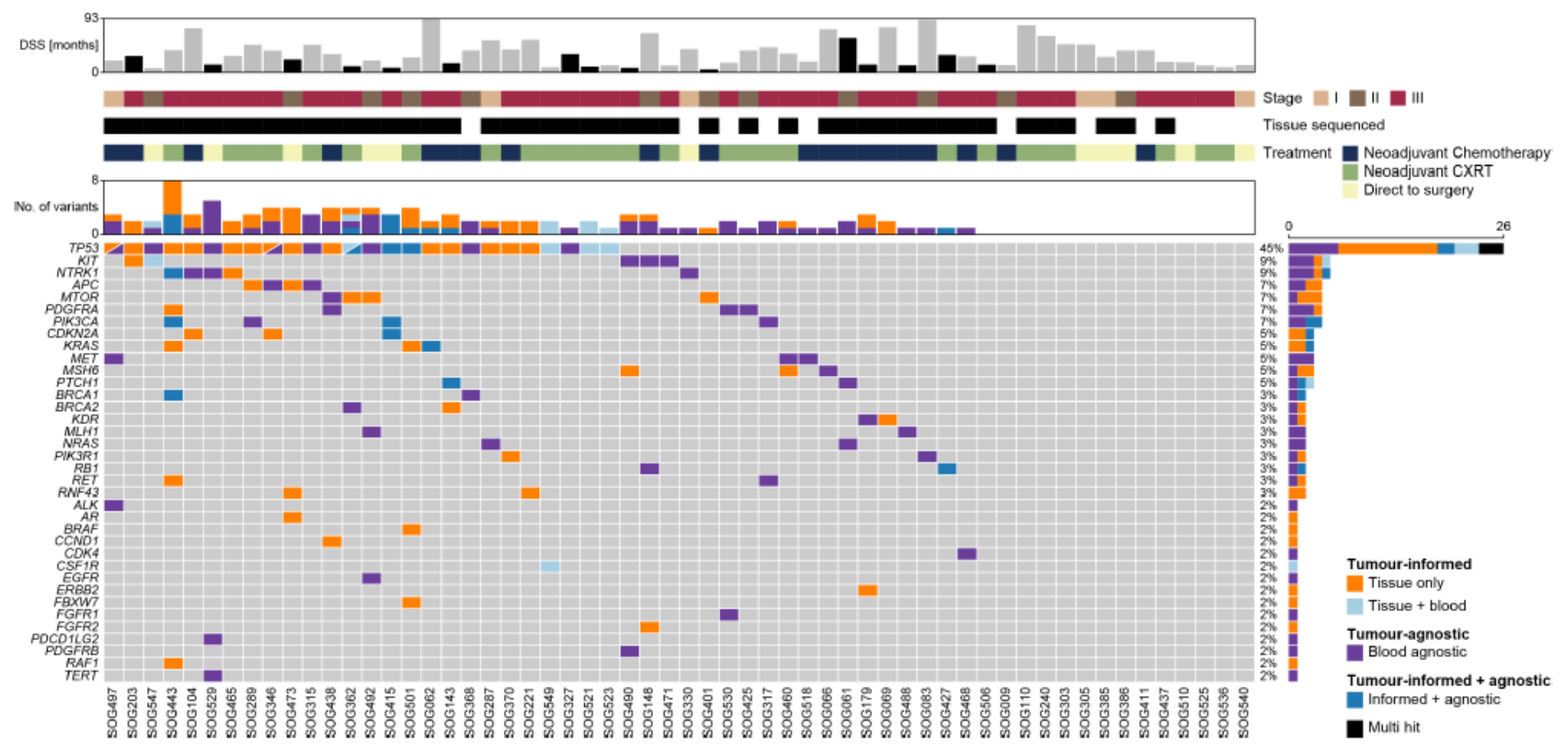

Figure 3

Variants detected in ctDNA and tumour DNA using a pan-cancer gene panel. The top panel shows disease specific survival (DSS) in months. Stage and treatment type are indicated. The number of variants per patient is shown, range 0 to 9 . Mutated genes are plotted for each patient. Overall, $45 \%$ of patients harboured a TP53 variant. The analysis approach is represented using a colour code (tumour informed or tumour agnostic approach). CXRT is chemoradiotherapy. 

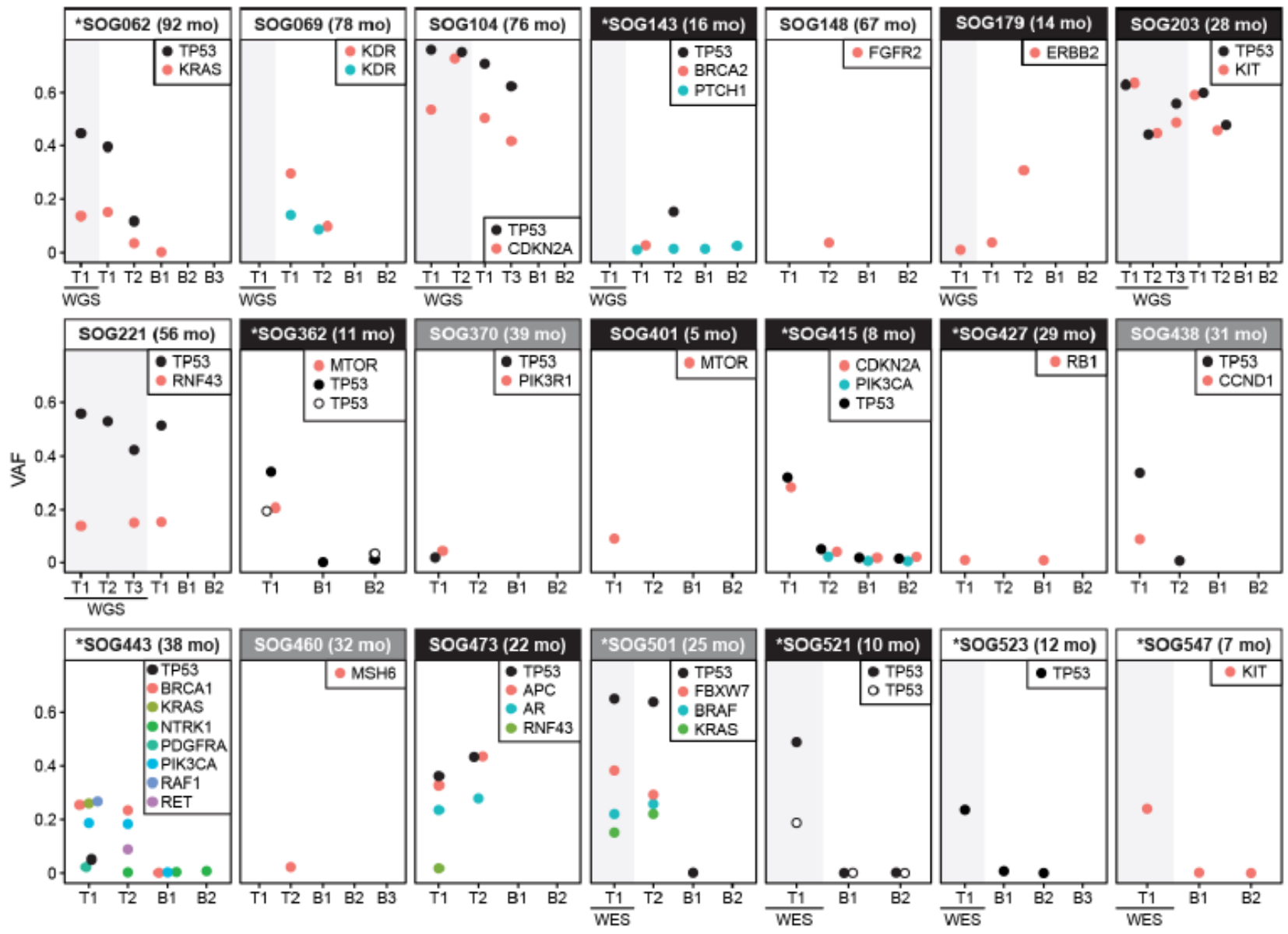

\section{Figure 4}

Overview of variants detected using a pan-cancer gene panel. Variant allele frequency (VAF) in tumour and blood samples analysed by whole-genome sequencing (WGS), whole-exome sequencing (WES) and the AVENIO tumour and ctDNA platform. Samples run using WGS or WES are indicated. SOG066, SOG083 and SOG506 are not represented as they had no tumour variants detected using any platform. Biopsies ( $\mathrm{T} 1, \mathrm{~T} 2$ and $\mathrm{T} 3$ ) were taken from multiple sites within the primary sample. Blood samples were taken at baseline, B1, and after treatment, B2 and B3. Patient disease specific survival is indicated in brackets. NSR is no sign of recurrence. The star indicates patients that are shedders. 

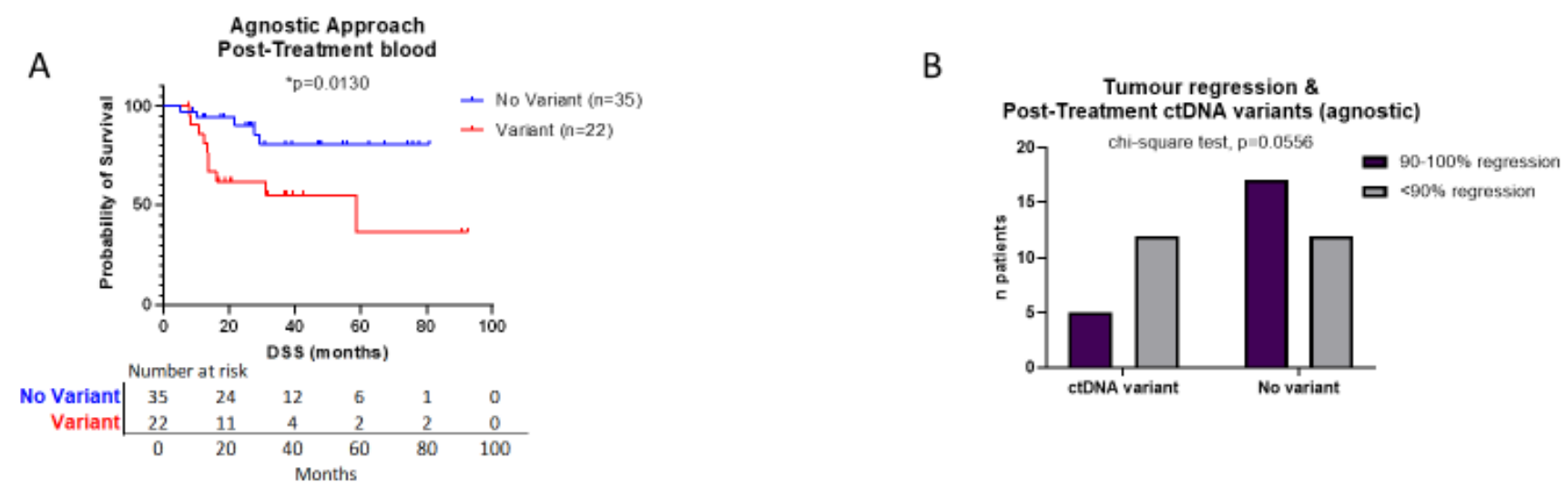

C
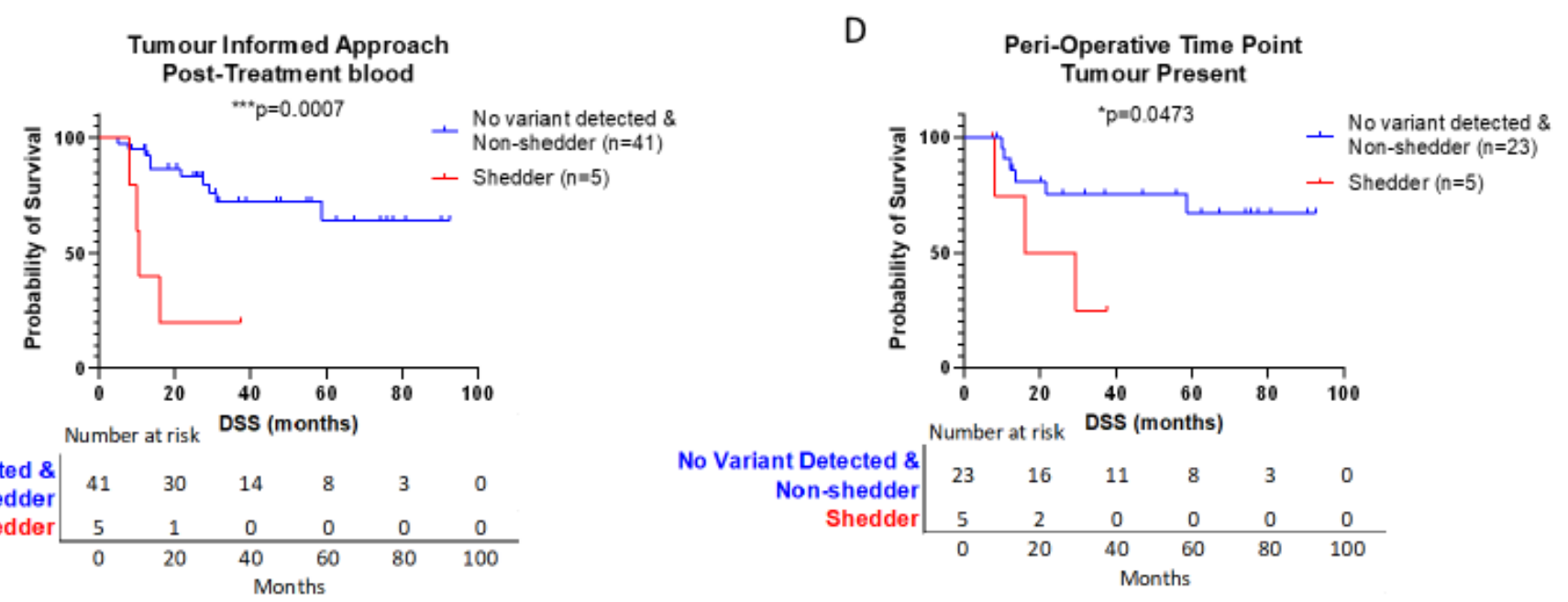

Figure 5

Prognostic significance of ctDNA in post-treatment blood samples. A. Disease specific survival (DSS) analysis in the tumour agnostic approach, showing patients with detectable ctDNA variants had worse survival ( $\left.{ }^{*} p=0.0130\right)$ B. Chi-square test assessing the correlation between major histological response and ctDNA variants in post-treatment bloods, agnostic approach $(p=0.0556) \mathrm{C}$. DSS analysis in the tumour informed approach assessing the post-treatment blood samples $\left({ }^{\star \star *} p=0.0007\right) \mathrm{D}$. Analysis at the peri-operative time point in patients with a measurable primary tumour using a tumour informed approach $\left({ }^{*} p=0.0473\right)$. Patients described as shedders have ctDNA variants that are confirmed in the primary tumour. Non-shedders have primary tumour variants that are not detected in the blood samples. No variant detected indicates an absence of primary tumour variants. 

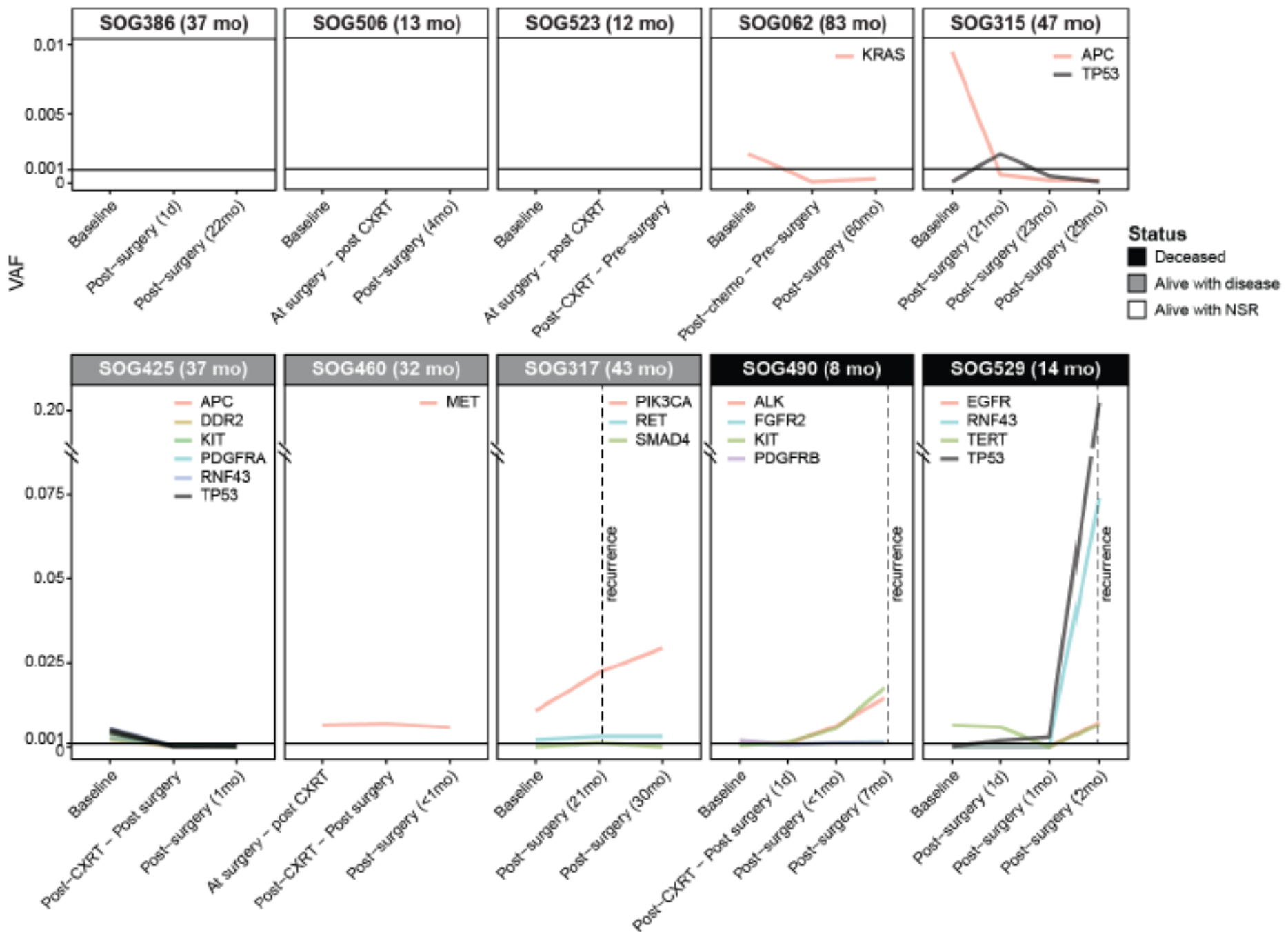

Figure 6

Serial Time Point Analysis of plasma derived ctDNA variants. Variant Allele Frequency (VAF) of ctDNA detected across serial time points for 10 patients. The threshold for variant calling is 0.001 , represented by the black horizontal line. Disease specific survival is indicated for each patient in brackets. Recurrence time is indicated by the dotted line. NSR is no sign of recurrence. Clinical time point is described where chemo is chemotherapy and CXRT is chemoradiotherapy.

\section{Supplementary Files}

This is a list of supplementary files associated with this preprint. Click to download.

- ctDNAOACSupplementaryFigures.pdf

- ctDNAOACSupplementaryTable1.xlsx 